\title{
What a successful grasp tells about the success chances of grasps in its vicinity
}

\author{
Leon Bodenhagen, Renaud Detry, Justus Piater and Norbert Krüger
}

\begin{abstract}
Infants gradually improve their grasping competences, both in terms of motor abilities as well as in terms of the internal shape grasp representations. Grasp densities [3] provide a statistical model of such an internal learning process. In the concept of grasp densities, kernel density estimation is used based on a six-dimensional kernel representing grasps with given position and orientation. For this so far an isotropic kernel has been used which exact shape have only been weakly justified. Instead in this paper, we use an anisotropic kernel that is statistically based on measured conditional probabilities representing grasp success in the neighborhood of a successful grasp. The anisotropy has been determined utilizing a simulation environment that allowed for evaluation of large scale experiments. The anisotropic kernel has been fitted to the conditional probabilities obtained from the experiments.

We then show that convergence is an important problem associated with the grasp density approach and we propose a measure for the convergence of the densities. In this context, we show that the use of the statistically grounded anisotropic kernels leads to a significantly faster convergence of grasp densities.
\end{abstract}

\section{INTRODUCTION}

When using already made grasping experience with a specific object there is no way to repeat the exactly same grasp due to uncertainties on pose estimation as well as the actual grasping process as such. Hence assumptions about grasps likely to be successful in the vicinity of grasps already tested and memorized are required even when exactly the same grasp is repeated for the same object in a new situation.

To tackle this, recently the concept of grasp densities [3] has been introduced which describes grasp affordances associated with specific objects in a probabilistic way. It is based on a kernel density estimation [9] in which the success likelihood of already tried grasps is described by 6-dimensional kernels (illustrated in figure 1 bottom left). The set of all grasp affordances associated with an object can then be expressed as the sum of these spatially extended 6D kernels (see figure 1 top right). Grasp densities have proven very useful in applications since they allow for the formulation of optimal grasps under constraints (see figure 2). In addition, since grasp densities reflect success likelihoods of each grasp, these can be used in higher level processes such as action sequence planning.

L. Bodenhagen and N. Krüger are at University of Southern Denmark. Email: lebo@mmmi.sdu.dk

R. Detry is at the Royal Institute of Technology, Sweden

J. Piater is at the University of Innsbruck, Austria
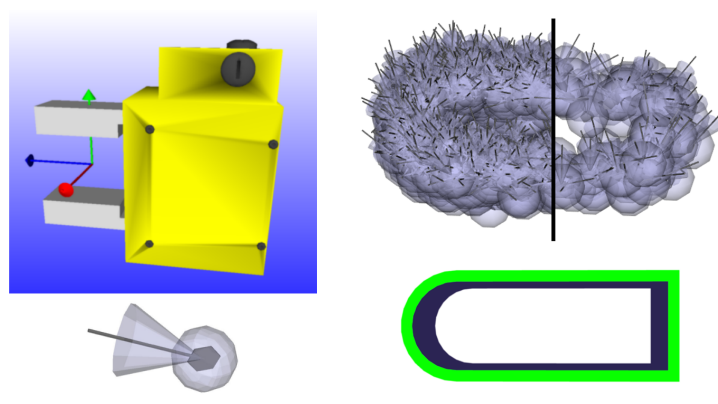

Fig. 1: Illustration of the parallel-jaw gripper (top left) and the 6D-kernel associated with the grasp (bottom left). And a visualization of a grasp density associated with an artificial object (top right). The left part shows all kernels, the right part only $10 \%$ of the kernels in order to provide a better overview. The original object is shown at the bottom (right).

However, currently two severe problems exist when using grasp densities. First, the exact shape of the kernels being used in previous work is only weakly motivated and is in particular isotropic (see figure 1 bottom left). Nonetheless high structural dependencies can be assumed to exist in the space of grasps associated with objects due to the intrinsic regularities of objects. The understanding of these regularities and how they can be expressed in kernels is an interesting topic in itself. In this context the first contribution of our paper is to give a statistical justification of the shape of the success likelihood of grasps in the vicinity of already successfully tested grasps. This is done by means of statistically derived conditional probabilities in grasp simulations.

As we will show in this paper, a second problem of the grasp density approach [3] is a rather slow convergence of the algorithm when the complete set of affordances is supposed to be represented as it requires a large set of grasp attempts. In this paper we give evidence that by using the statistically derived anisotropic kernel, we can speed up the convergence of the algorithm significantly.

The paper is structured as following. In section II, we introduce basic notations and methods used in this paper. In section III, we introduce the results on our statistics which motivate the choice of a new anisotropic kernel. The adaption of the new anisotropic kernel to the statistical results is outlined in section III 


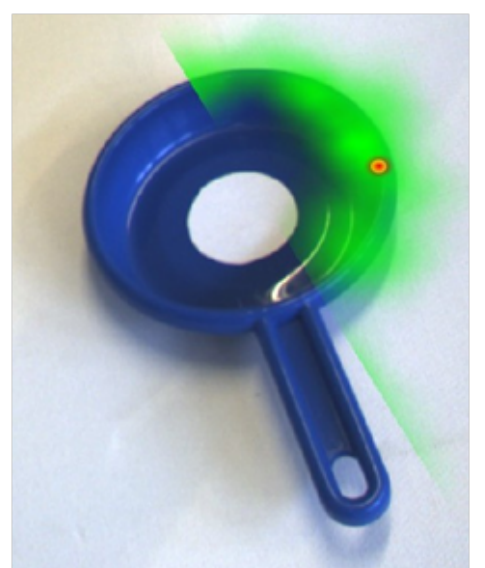

Fig. 2: Grasp success likelihoods learned by means of exploration are represented in green. A local maximum indicates optimal grasp points. Constraints of graspability (e.g., on invoked by workspace constraints as indicated by the sharp green border) can be easily integrated.

as well as its application to grasp density estimation.

\section{Methods}

In the following we provide a detailed overview of the different methods used throughout this paper. In section II-A our parametrization of grasping actions is defined, the association from actions to objects using grasp densities is described in section II-B. Two different designs for anisotropic kernels are outlined in section II-C. The simulation environment wherein actions are performed is introduced in section II-D and in section II-E the choice of bandwidths for an isotropic kernel is discussed.

\section{A. Grasps and their transformations}

A grasping action, $A$, is in this context defined as a point in the special Euclidean space, $A \in S E(3)$ and defines the transformation from the object reference frame to the tool which performs the grasp. In this work, the tool is considered to be a parallel finger gripper - the setup is illustrated in Fig. 3.

Based on a set of evaluated and successful grasps, $\mathcal{S}=\left\{A_{1}, \ldots, A_{n}\right\}$, for one specific object it is investigated if a grasp still would be successful when it becomes transformed locally:

$$
P\left(T_{R B M}(A) \text { is successful } \mid A \text { is successful }\right)
$$

where $T_{R B M}(x)$ denotes a rigid body motion applied to the action $A$, thus

$$
T_{R B M}: S E(3) \rightarrow S E(3)
$$

Note that $A$ and $T_{R B M}$, although both elements of $S E(3)$ have two separate meanings, $A$ representing a grasp and $T_{R B M}$ a rigid transformation.

The success of a transformed action, $T_{R B M}(A)$, is estimated by comparing it with all grasps in $\mathcal{S}$ using the grasp density (introduced in section II-B). To be able to do a reasonable comparison it is required that the density covers the entire object. It is not feasible to evaluate every $T_{R B M}(A)$ physically or even in a simulator as this still would be far too time consuming.

\section{B. Grasp Densities}

A grasp density models the distribution of successful grasps relative to an object. A density is defined as a probability density function, $p_{X \mid O=s}(x)$, where $X \in$ $S E(3)$ represents a gripper pose and $O \in\{s, f\}$ is the outcome of a grasp which can be either success or failure. The value of a grasp density at a concrete pose $x \in S E(3)$ is proportional to the likelihood of a successful grasp when the gripper is moved to this pose and closed. A typical applications for grasp densities is for example the search for a local maximum which provides the user with a grasp hypothesis with a high likelihood of being successful (see figure 2). External constraints, e.g. due limited workspace of a robot, can easily be integrated by limiting the search space (see figure 2).

A grasp density $d(x)$ is estimated using kernel density estimation (see also [2], [9]):

$$
d(x)=\sum_{i=0}^{n} w_{i} \mathbf{K}_{\mu_{i}, \sigma}(x)
$$

where $w_{i}$ is a weight that compensated the impact of the sampling strategy. The kernel $\mathbf{K}$ is defined as a product of a trivariate Gaussian kernel, $\mathbf{N}$, for the position and a orientation kernel $\Theta$ defined on $S O(3)$ by the von-Mises-Fisher distribution [5] (see also figure 1):

$$
\mathbf{K}_{\mu, \sigma}(x)=\mathbf{N}_{\mu_{t}, \sigma_{t}}(\lambda) \boldsymbol{\Theta}_{\mu_{r}, \sigma_{r}}(\theta)
$$

where

$$
\begin{aligned}
\Theta_{\mu_{r}, \sigma_{r}}(\theta) & =\frac{1}{2} C_{4}\left(\sigma_{r}\right)\left(e^{\sigma_{r} \cos (\beta)}+e^{-\sigma_{r} \cos (\beta)}\right) \\
\beta & =\cos ^{-1}\left(\mu_{r}^{T} \theta\right)
\end{aligned}
$$

where $\sigma_{t}$ denotes the width of the kernel for the position and $\sigma_{r}$ denotes the width of the kernel for the orientation. Similar $\mu_{t}$ and $\mu_{r}$ denote the mean values in $S E(3) . \lambda$ and $\theta$ are the pure positional respectively orientional part of $x=(\lambda, \theta)$ - orientations are in all cases represented using quaternions. $C_{4}\left(\sigma_{r}\right)$ is a constant which ensures that the density integrates to one. Note that $\boldsymbol{\Theta}_{\mu_{r}, \sigma_{r}}(\theta)$ basically depends on the angle between two quaternions which is a scalar (for further details, see [3]).

\section{Anisotropic kernels}

One property of the kernel $\mathbf{K}$ as introduced in equation 2 and in more detail in [3] is that both the position and the orientation are modeled isotropically (see figure 1a). Different strategies to model an anisotropic kernel, $\hat{\mathbf{K}}$, which expresses the structural properties of successful grasps in a neighborhood have 
been considered. Note that an anisotropic kernel can be defined as a sum of multiple isotropic kernels with constant widths:

$$
\hat{\mathbf{K}}_{\mu, \sigma}^{S}(x)=\sum_{j=0}^{m} w_{j} \mathbf{K}_{\mu_{j}, \sigma_{j}}(x)
$$

allowing for an approximation $\hat{d}(x)$ of $d(x)$ by

$$
\hat{d}(x)=\sum_{i=0}^{n} w_{i} \hat{\mathbf{K}}_{\mu, \sigma}^{S}(x)
$$

Although the approach in equation (5) is computationally not optimal it can be used to investigate the impact of using anisotropic kernels for the generation of grasp densities and it does not imply any restriction on the shape of the anisotropic kernel.

The alternative is an analytic expression of $\hat{\mathbf{K}}_{\mu, \sigma}(x)$. Remembering that the value of original kernel used for the orientation is dependant on the angle between two quaternions (see equation 3). This angle can be weighted which leads to the following formulation of the kernel:

$$
\begin{aligned}
\hat{\boldsymbol{\Theta}}_{\mu_{r}, \sigma_{r}}(\theta)= & \frac{1}{2} C_{4}\left(\sigma_{r}\right)\left(e^{\sigma_{r} \cos \left(w_{\mu_{r}}(\theta) \beta\right)}\right)+ \\
& \frac{1}{2} C_{4}\left(\sigma_{r}\right)\left(e^{-\sigma_{r} \cos \left(w_{\mu_{r}}(\theta) \beta\right)}\right)
\end{aligned}
$$

where the angle is weighted by $w_{\mu}(\theta)$, which is similar to Mahalanobis distance measure [8]:

$$
w_{\mu_{r}}(\theta)=\sqrt{(\theta-\mathbf{0}) S^{-1} R_{\mu_{r}}(\theta-\mathbf{0})^{\prime}}
$$

where $S$ is a diagonal matrix that describes a 4Dellipsoid and $R_{\mu_{r}}$ represents the rotation that aligns the ellipsoid with the mean-orientation of the kernel. Similarly the Gaussian kernel $\mathbf{N}$ can be defined using a covariance matrix to use the Mahalanobis distance rather than the Euclidean. In this paper we describe the basis for such an approximation as outlined in the discussion.

\section{Simulator and Simulations}

As a large set of evaluated grasps is required to achieve complete coverage of grasp affordances a simulator [7] has been used (in total we simulated about 10.000.000 grasps). The setup is illustrated in figure 3 . The use of a simulator allows us to evaluate large sets of grasps efficiently while avoiding the overhead of using a real setup and circumventing the introduction of errors by usage of a pose estimation algorithm to obtain the pose of the object.

In the simulator both objects and grippers are defined by geometric models as well as mass and friction information. While the material of the gripper is known, we estimate the mass of the objects and assume them to be made of plastic. For the gripper it is in addition ensured that the realistic constraints on the position, velocity and acceleration of the fingers are maintained.

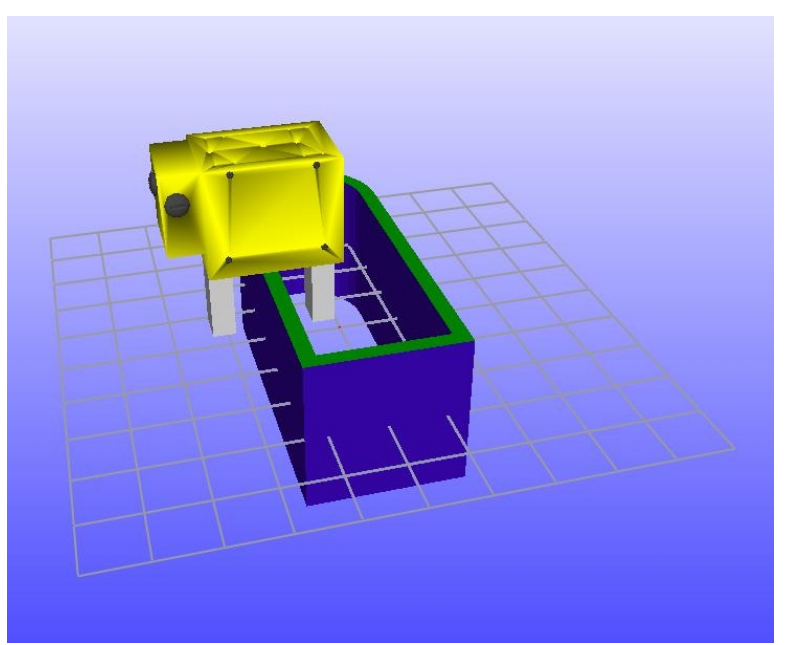

Fig. 3: Screenshot from the simulator.

The positions of the grasps that are to be simulated are obtained by defining a $6 \mathrm{D}$ grid covering all the poses that are in the vicinity of the object. The resolution of this grid needs to be limited in order to ensure that simulation still is tractable. In our simulation, we used a grid of $10 \mathrm{~mm}$ and approximately 15 degrees.

The simulator models the interaction between the tool, a simulated Schunk PG70 parallel-jaw gripper with a maximum finger distance of $70 \mathrm{~mm}$, and the object during the grasping process using a physics engine. The execution of a grasp is finished when either the gripper is closed entirely or the object prevents it from doing so (see figure 3). Grasp hypotheses that would lead to collisions between gripper and object beforehand are discarded. Whether a grasp is successful or not is estimated by calculating the grasp force wrench and determining whether the grasp can counteract gravity (see also [4]). If that is the case, the grasp is considered to be successful. We only consider the force wrench as the simulation is based on hard contacts. Therefore a stable grasp may consist of only two contact points, although the object might rotate freely on the axis defined by the two contact points.

The four different objects used in our simulations are shown in figure 4 . The screwdriver and the coffee-mug have been found on the web ${ }^{1}$. Their size matches the size of common real world objects. The screw driver is approx. $280 \mathrm{~mm}$ long and up $30 \mathrm{~mm}$ wide, the body of the cup is approx. $60 \mathrm{~mm}$ wide and $80 \mathrm{~mm}$ high. The elongated D-shaped object (top right in figure 4) and the cone are purely artificial objects. The width of the cone is $80 \mathrm{~mm}$ at it's base and $20 \mathrm{~mm}$ at the top while the overall length is $400 \mathrm{~mm}$. The dimensions of the elongated D are approx. $140 \mathrm{~mm} \times 140 \mathrm{~mm} \times 350 \mathrm{~mm}$.

Figure 5 shows a visualization of a grasp density that has been created for the cone. For the projections of the density a plane has been defined that contains the main axis of the cone and is parallel to the image

\footnotetext{
${ }^{1}$ http://sketchup.google.com/3dwarehouse/
} 


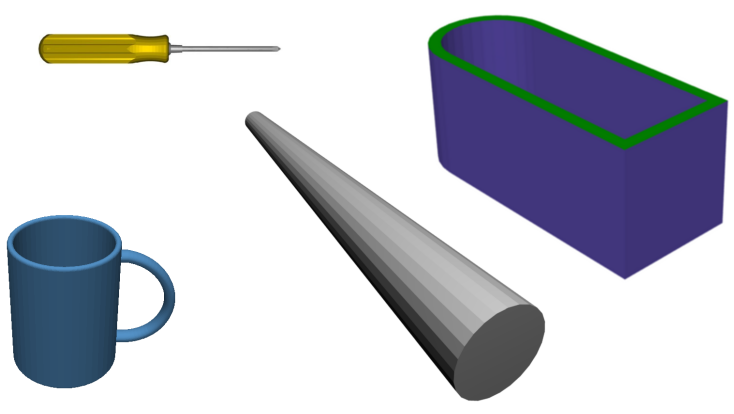

Fig. 4: The different objects used for the simulations. Some of the objects have been scaled for illustrative purposes.

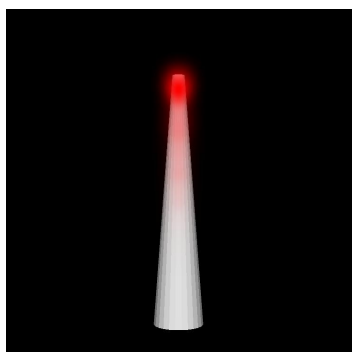

(a) (b)

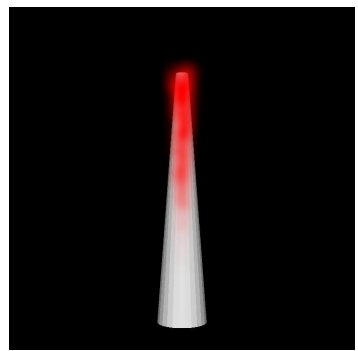

Fig. 5: An example of a grasp density. The more opaque the red color is, the higher the values of the density is at this point. (a) illustrates the distribution of all grasps, (b) illustrates the distribution of all grasps that are oriented vertical to the image plane and aligned with the main axis of the object.

plane of a virtual camera. Subsequently the density has been projected on the plane in red. The opaqueness of the color indicates the value of the density where completely opaque refers to the maximum and completely transparent regions indicate that no successful grasps have been experienced there. Note that the projections have been normalized individually to guarantee that the highest value of projection saturates the red colore channel. In figure 5a all grasps have been projected showing that most successful grasps occurred at the narrow end of the cone. Figure 5b show the distribution of all grasps that are oriented vertical to the image plane and aligned with the main axis of the object.

\section{E. Optimal isotropic kernels}

The grasps obtained using the simulator are used to create a grasp density. This process requires that a suitable kernel width is selected. There is a trade-off between having small kernels requiring a fine-grained density using a large number of kernels to reach a full coverage but allows to have relatively sharp borders separating successful and non successful grasps and having wide kernels which ensure that entire graspable part of the object is covered by the density using fewer samples with the cost that borders between successful

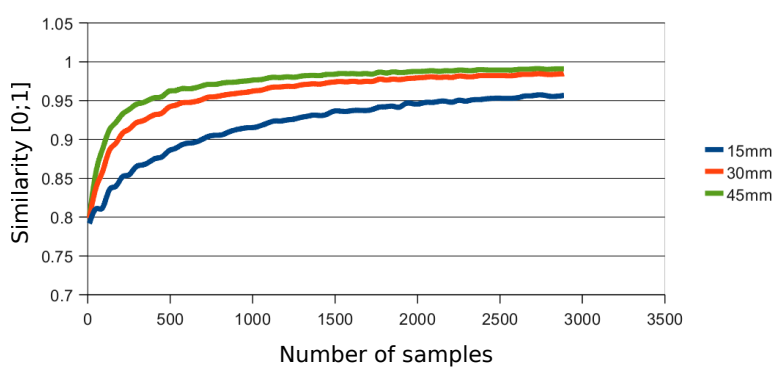

Fig. 6: Bootstrapping applied to a density using three different kernel widths.

and non-successful grasps are blurred. In this section we discuss this trade-off aiming at estimating a suitable kernel width.

Since the set of true grasp affordances is not directly accessible, it is difficult to select proper widths. Therefore the concept of bootstrapping (see [6]) is utilized in order to achieve a convergence measure $s$. Bootstrapping is in general a strategy to estimate statistical properties of any measurement by estiming these properties directly on samples of the distribution that approximates the measurement. The measurement in our case is the overall graspability of the object, estimated by a grasp density. Each grasp is considered to be a sample of the overall graspability. Given a grasp density based on $N$ samples, $N$ new samples are drawn randomly with replacement and a new density is created based on these samples. This procedure is repeated $B$ times and the similarity $s$ of the $B$ resampled densities is estimated:

$$
s=\frac{1}{B} \sum_{b=1}^{B} B C\left(d_{\mu}(x), d_{b}(x)\right)
$$

where $B C()$ denotes the Bhattacharyya Coefficient [1] and $d_{\mu}(x)$ is the mean density over the $B$ sets. Given a large value for $B, d(x)$ is used as an approximation of $d_{\mu}(x)$ in equation (9). Note that although $s$ is defined similarly to the variance, the variance approaches zero when $s$ approaches the value of one, which is interpreted as the density being fully converged.

The more the individual kernels of a density overlap, the smaller the variance of the re-sampled densities is expected to be and as a consequence the similarity $s$ will approach 1. Figure 6 shows the convergence of three densities which are based on the same samples but using different widths. Although it is obvious that the larger kernel leads to a faster convergence, it is important to keep in mind that the densities do not converge to the very same density as each larger kernel covers a larger region, even though this does not necessarily reflect the true grasp density. When samples are drawn randomly (and not obtained by the search for a maximum ) from the final densities, the average success ratios of the samples have been found to be $9.76 \%(45 \mathrm{~mm}), 18.18 \%(30 \mathrm{~mm})$ and $30.08 \%(15 \mathrm{~mm})$. Note that these numbers do present 


\begin{tabular}{|l|rrr|}
\hline Object & successes & failures & ratio \\
\hline Cone & 166.690 & 3.331 .043 & $5.0 \%$ \\
Elongated D & 2.898 & 99.764 & $2.9 \%$ \\
Screwdriver & 159.947 & 1.574 .709 & $10.1 \%$ \\
Mug & 19.051 & 4.127 .861 & $0.46 \%$ \\
\hline
\end{tabular}

TABLE I: Overview over the number of successful and failing grasps for the individual objects and the success ratio.

results in a very sub-optimal use of grasp densities in which also grasps are tested which are known to have a low success likelihood (i.e., where the grasp density has low values). This can be very useful, when we want to explore grasps corresponding to areas in $S E(3)$ where there are unstable grasps. It is a quality of the grasp density also to represent these kind of areas appropriately. However, one needs to be aware of the trade-off discussed here.

\section{RESULTS}

The estimated conditional probabilities $P\left(T_{R B M}(A)\right.$ is successful $\mid A$ is successful $)$ of the success of the displaced successful grasps for various objects is given in section III-A. Results in the context of the adaption of an anisotropic kernel to these statistics is outlined in section III-B. results on the convergence using this anisotropic kernel are given in section III-C.

\section{A. Statistical Results}

A feasible method for an investigation of the results of the statistical investigation are multidimensional histograms where one axis covers the likelihood of a grasp to be successful and each direction of displacement leads to an additional axis. As it is hard to visualize high-dimensional histograms, only two dimensions of displacements will be covered in a single histogram and the mean success likelihoods of the grasps associated with the individual bins are computed.

Given an object that can be grasped at its edge, it is expected that successful grasps can be translated along the edge (see figure $7 \mathrm{a}$ where the green marker represents a successful grasp and the orange ones represent grasps we would expect to succeed as well). However, grasps will also be translatable on the two orthogonal directions depending on the finger-width of the gripper (in conjunction with the thickness of the edge) as well as the length of the fingers. Further it is expected that grasps can be rotated around the axis defined by the normal of the surface ending at this edge (illustrated in figure 7b). Figure 7c indicates the associated coordinate system.

The numbers of succeeding and failing grasps for the different objects are listed in table I. Note that the success likelihood is very different for the different objects. The screwdriver is relatively easy to grasp, even a random grasp has a success chance of more
(1) (2) (3)

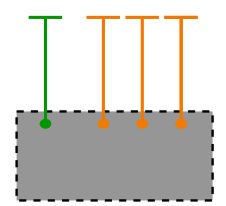

(a)

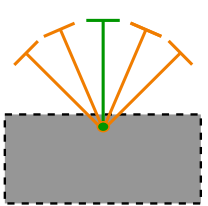

(b) (c)

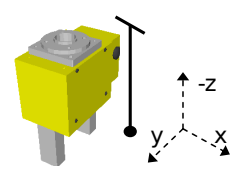

Fig. 7: (a) and (b):Given that the green grasps (visualized in 2D) have been found to be successful and the gray box represents the grasped object, it is expected that the orange grasps would be successful as well. (c) Illustration of the gripper (1), a simplified view (2) and the 3 axes of the associated frame (3).

than $10 \%$. For the 'Elongated D' a random grasp has a probability of success of less than $3 \%$ while for the the cone the success likelihood is $5 \%$. For the mug a random grasp has a very low success chance of only $0.5 \%$. Note that for the mug only few grasps will succeed since there are only few graspable positions, and these need to be approached with carefully aligned grasp orientation. For each object an individual grasp density has been created based on the successful grasps (see, e.g., figure 5). Note that the kernel $\mathbf{K}$ does not handle the fact that the gripper is mirror-symmetric around its approach axis. Therefore each sample is used to create the density both unaltered and rotated 180 degrees around the approach-axis of the gripper (the Z-axis, illustrated in figure 7c).

Subsequently each successfully tried grasp is used to generate a set of samples in its vicinity. For each of these samples the grasp density associated with the object is used to estimate the likelihood of graps corresponding to the new sample to be successful. As it is intractable to sample the complete $6 \mathrm{D}$ neighborhood for every tried grasp, a kernel is defined at the location of a tried grasp. Subsequently this kernel is sampled. Thereby the entire neighborhood of grasps can be covered, without exhausting it with every single tried grasp.

The average success likelihoods of the transformed grasps relative to the transformation (i.e., $P\left(T_{R B M}(A)\right.$ is successful $\mid A$ is successful $\left.)\right)$ for the object 'Elongated D' is shown on the histograms in figure 8 . The color of each bin reflects the successlikelihood of the grasps, 0 at the scale indicate $0 \%$ success likelihood, 1 indicates $100 \%$.

The histograms in the top row cover the translations - each histograms covers the translations in the $\mathrm{X}$ and $\mathrm{Y}$ - axis (horizontal resp. vertical axis on the histogram). Further, each histogram is accumulated over a range of displacements of $16 \mathrm{~mm}$ in the $\mathrm{Z}$-axis $(-24 \mathrm{~mm}$ to $-40 \mathrm{~mm}$ in the leftmost histogram, $24 \mathrm{~mm}$ to $40 \mathrm{~mm}$ in the rightmost). Each histogram in the bottom row covers two axis of rotations. The relation between a grasp and the different axes is shown in 

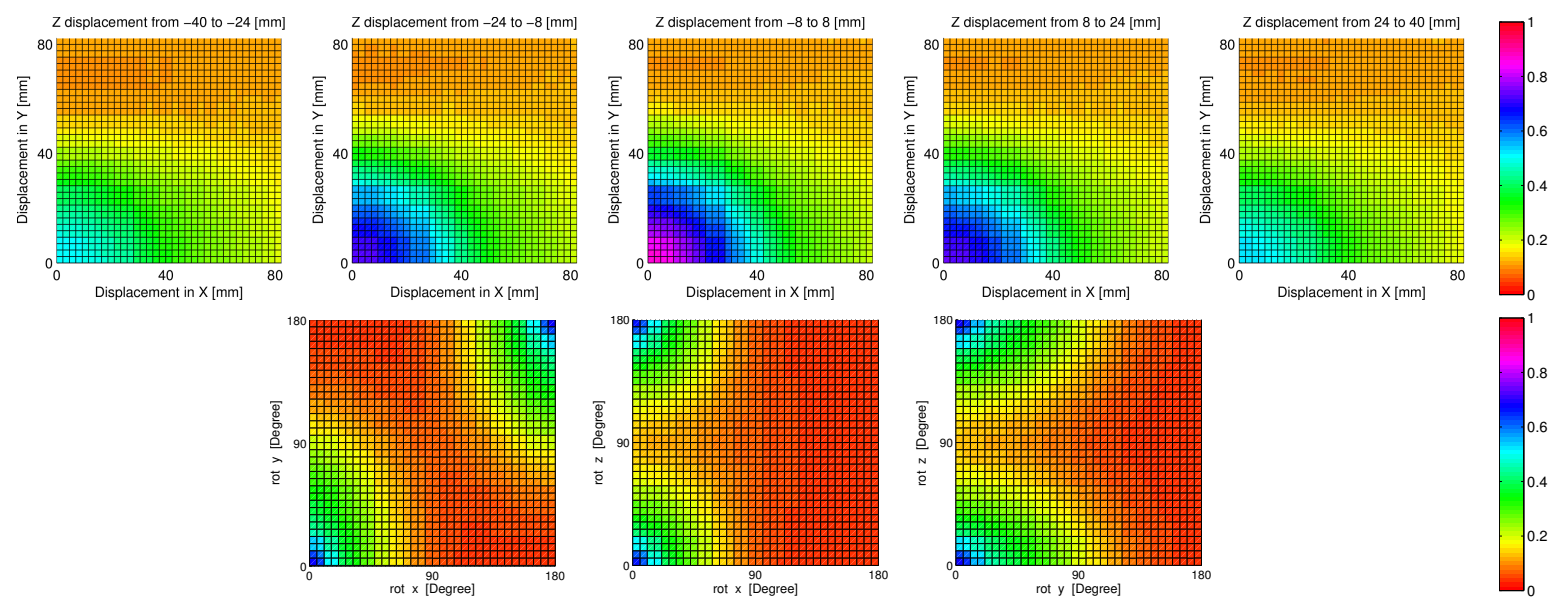

Fig. 8: Results for the object 'Elongated D' for both rotation and translation using the grasp densities with $30 \mathrm{~mm}$ wide kernels for position.

figure $7 \mathrm{c}$.

Results indicate that successful grasps are more robust towards translation in $\mathrm{x}-$ and $\mathrm{z}$-direction and rotations around their $y$-axis than the two remaining axes. This confirms our expectations visualized in figure 7. Note that when rotations are applied symmetries become explicit (see bottom row in figure 8 ). When a grasp for instance is rotated 180 degrees around both it's $\mathrm{x}$ - and $\mathrm{y}$-axis, the resulting configuration will be identical with the initial one, just mirrored around the $\mathrm{Z}$-axis which is identical with the approach-vector of the tool. Due to symmetry of the gripper the resulting grasp is considered to be identical with the initial one.

However, the results are not as explicit as one might have expected. It can be observed that a wide range of different orientations may still lead to a successful grasp at the very same position. Hence grasps do not have to be aligned with the edge of the object in order to be successful. But when the grasp and the object are not aligned, the translations we apply are not aligned with the object either and will lead to likely to be unsuccessful grasps. This becomes evident when we look at the corresponding statistics when we align the kernel with the main orientation of the object (see figure 9): It can be seen that the anisotropic structure is much more expressive both in translation and orientation. As a consequence of this investigations we can conclude that it is important to align an anisotropic kernel with the visually extracted edge shape structure.

In figure 10 - 12 we see the analog statistics (only the two center sub-figures corresponding to figure 9 and 8 are displayed). As we can see, the results for the screwdriver (figure 10) and the cone (see figure 12) look similar to the object 'Elongated D'. However, the structure of the conditional probabilities is different for the mug (figure 11). The reason is that the mug has only highly curved edges for which only slight translations of successful grasps lead to errors. Here also an alignment to the actual curved object shape would be appropriate.

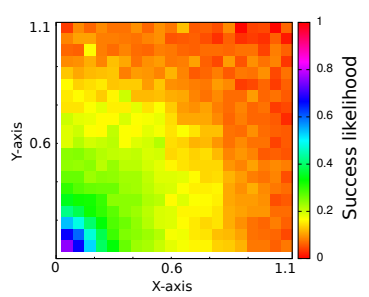

(a) Rotations (aligned axes)

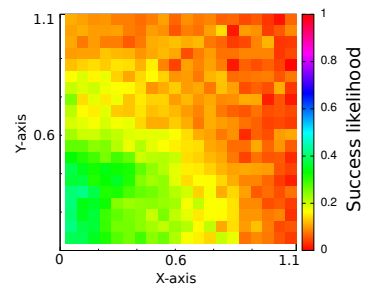

(c) Rotations (original axes)

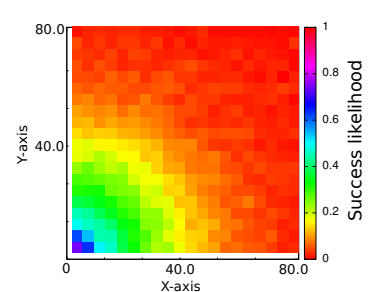

(b) Translations (aligned axes)

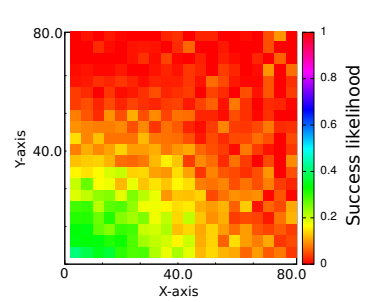

(d) Translations (original axes)
Fig. 10: Histograms for the screwdriver

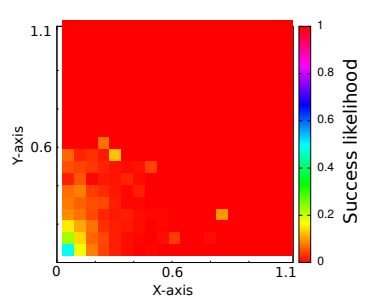

(a) Rotations

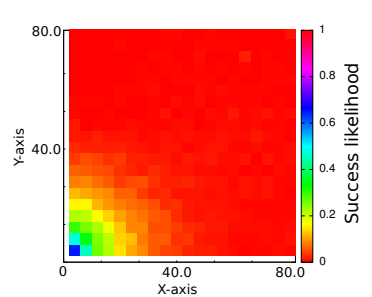

(b) Translations
Fig. 11: Histograms for the mug (not aligned) 

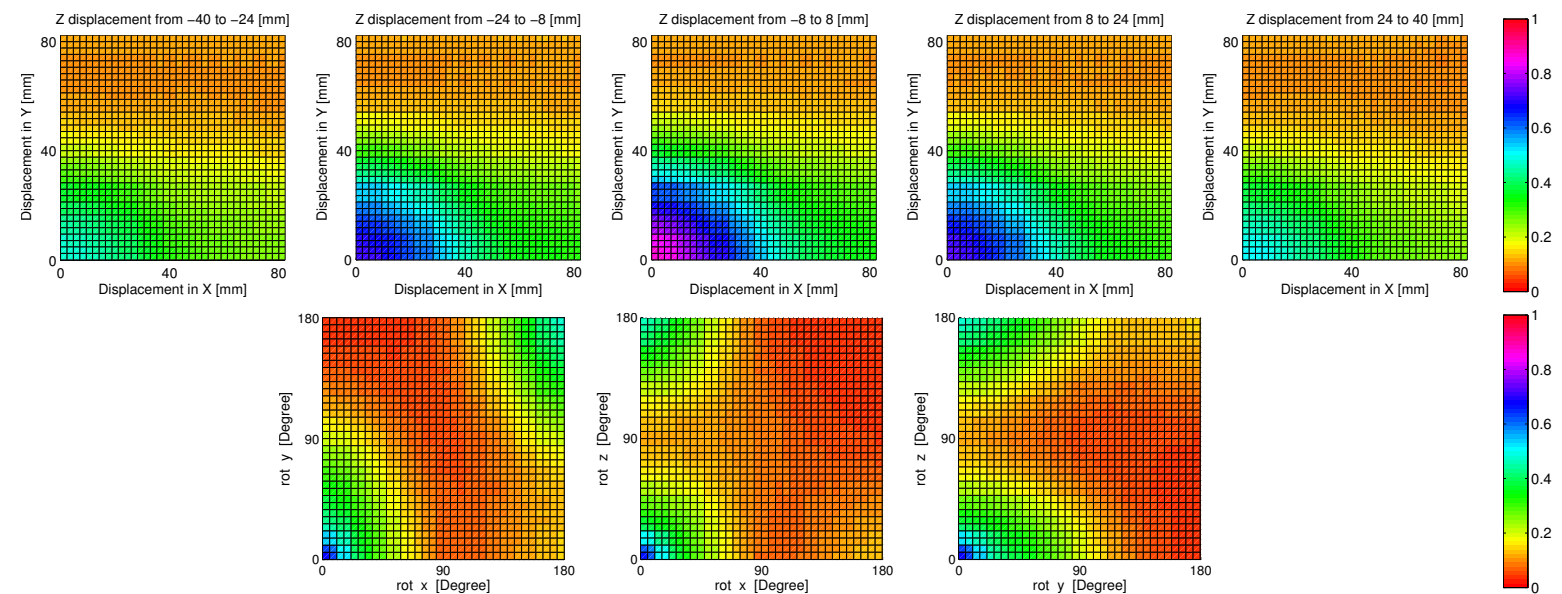

Fig. 9: Results for the object 'Elongated D' when the grasps are translated aligned with the object structure.

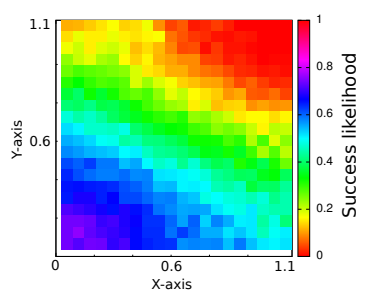

(a) Rotations (aligned axes)

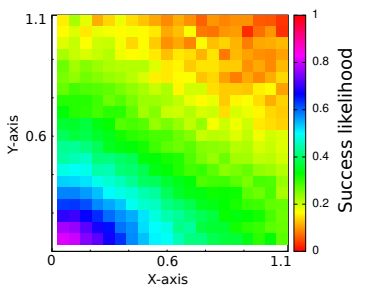

(c) Rotations (original axes)

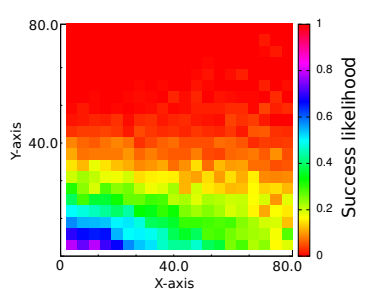

(b) Translations (aligned axes)

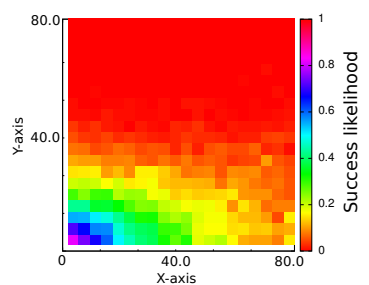

(d) Translations (original axes)

Fig. 12: Histograms for the cone

\section{B. Adapted kernel}

When using the definition of a kernel given in equation (5) the anistropic kernel is defined as a sum of isotropic kernels. This sum of kernels can directly be obtained by drawing are sufficiently large number of samples from the results that form the basis for the histograms in section III-A. Figure 13 illustrates the similarity between the results used for the histograms and the anisotropic kernel with respect to the number of samples used for the kernel estimation. The results show that a rather high number of samples are required in order to achieve estimate of the anisotropich kernel. In order to limit the computational costs we limit the kernel to consist of 900 samples which leads to a similarity of approximately 0.8 , estimated using the Bhattacharyya Coefficient [1].

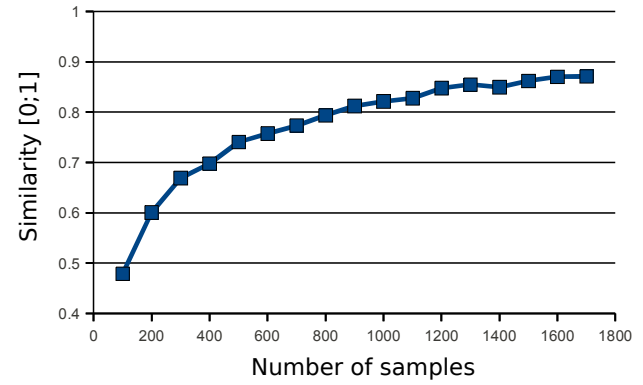

Fig. 13: Comparing the sampled kernel with the full data set used for the histograms for different numbers of samples.

\section{Convergence}

The usage of the anisotropic kernel does not imply significant changes to the learning of a grasp density. Independent on the type of the kernel, one kernel is added to the density every time a successful grasp is experienced. When the anisotropic kernel is used all kernels that it consists of are transformed according to the pose of experienced grasp and subsequently the composition of kernels is added to the density. Figure 14 shows a comparison of the convergence of two densities (both are based on the set of grasps learned for the elongated D), one using the anisotropic kernel and one using the isotropic kernel with a similar width. It becomes explicit the density that is using the anisotropic kernel converges significantly faster.

\section{CONCLUSION AND Discussion}

In this paper we have investigated the conditional probabilities of grasps in the vicinity of an already performed successful grasp for a number of objects. We found a large degree of structure in these conditional probabilities with large anisotropies. We have used these anisotropies to derive statistically justified kernels for grasp density estimation [3] and we have 


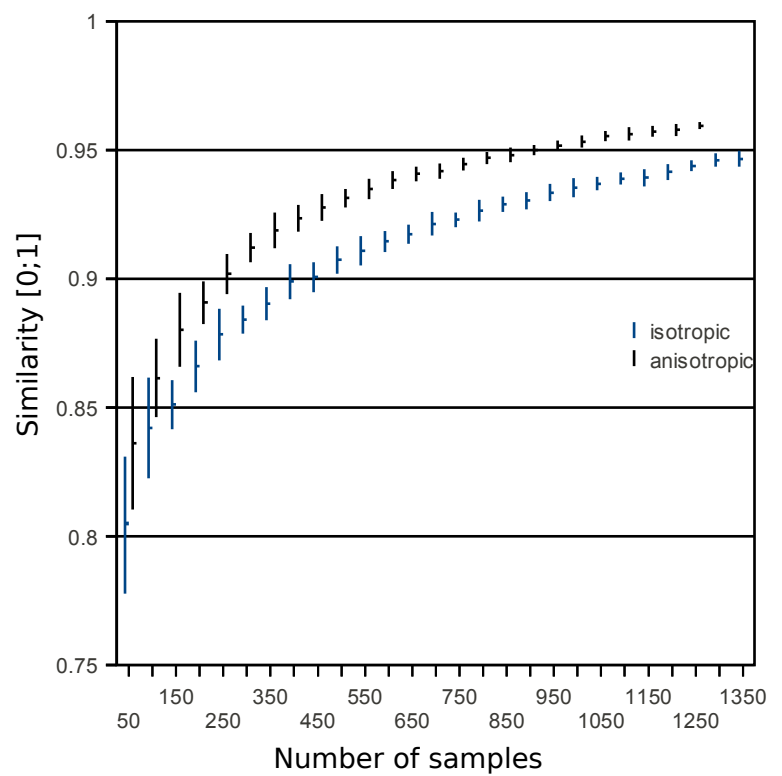

Fig. 14: Comparing convergence of the isotropic kernel and the anisotropic kernel. The vertical bars indicate the standard deviation of the individual similarity measurement.

shown that based on these kernels a faster convergence of grasp densities can be achieved. By that the statistical experience made during grasping can influence the actual learning approach on a meta level. We believe that the efficient use of such statistically derived conditional probabilities is one of the main reasons for successful development of cognitive agents. We have also shown that it is important to align the derived kernels with the actual structure of the object shape: Considering the scenario in [3] where local 3D edge-descriptors of the scene have been used to create a proposal grasp density describing potential grasps. We imagine that the introduction of anisotropic kernels allows us to utilize the edge information further. Rather than generating a proposal density consisting of a vast number of kernels, anisotropic kernels can be fitted to edge segments, thereby covering structural similar regions. As a consequence less samples are needed to formulate a proposal density and less samples are required to sample the entire object.

In future work we will finalize the experiments with the analytically defined anisotropic kernel. Furthermore we aim at comparing the simulation data with real robot data and to derive higher order conditional probabilities associated with more complex feature grasp associations such as coplanar and/or parallel edge and surface structures.

\section{ACKNOWLEDGMENTS}

This work was supported by the EU Cognitive Systems project XPERIENCE (FP7-ICT-270273).

\section{REFERENCES}

[1] A. Bhattacharyya. On a measure of divergence between two statistical populations defined by probability distributions. Bull. Calcutta Math. Soc., 1943. p. $99-109$.

[2] R. Detry, E. Başeski, N. Krüger, M. Popović, Y. Touati, O. Kroemer, J. Peters, and J. Piater. Learning object-specific grasp affordance densities. In Int. Conf. on Development and Learning, 2009.

[3] R. Detry, D. Kraft, O. Kroemer, L. Bodenhagen, J. Peters, N. Krüger, and J. Piater. Learning grasp affordance densities. Paladyn Journal of Behavioral Robotics, (accepted), 2011.

[4] C. Ferrari and J. Canny. Planning Optimal Grasps. In IEEE Int. Conf. on Robotics and Automation, pages 2290-2295, 1992.

[5] R. Fisher. Dispersion on a sphere. Royal Society of London. Series A, Mathematical and Physical Sciences, Volume 217 Issue 1130:295-305, 1953.

[6] R. W. Johnson. An Introduction to the Bootstrap. Journal of the Royal Statistical Society, 23:49-54, 2001.

[7] J. A. Jørgensen and H. G. Petersen. Usage of simulations to plan stable grasping of unknown objects with a 3-fingered Schunk hand. In IEEE Int. Conf. on Intelligent RObots and Systems (IROS), Workshop - Robot simulators: available software, scientific applications and future trends, 2008.

[8] P. C. Mahalanobis. On the generalised distance in statistics. Proceedings of the National Institute of Science of India, 12:49_ 55, 1936.

[9] B. W. Silverman. Density Estimation for Statistics and Data Analysis. Chapman and Hall/CRC, 1986. 